\title{
Crystal structure of $\mu_{2}$-hydroxyl-bis(5-methyl-1,4,9-triazanonanezinc(II)) triperchlorate, $\mathrm{C}_{14} \mathrm{H}_{39} \mathrm{Cl}_{3} \mathrm{~N}_{6} \mathrm{O}_{13} \mathrm{Zn}_{2}$
}

\author{
H.-L. Zhu*, F.-J. Meng, Z.-D. Wang and F. Yang \\ Wuhan Institute of Science and Technology, Department of Environmental and Chemical Engineering, Wuhan, 430073 P. R. China
}

Received March 17, 2003, accepted and available on-line June 25, 2003; CCDC-No. 1267/1072<smiles>O=C(C1CC1)C1CC1</smiles>

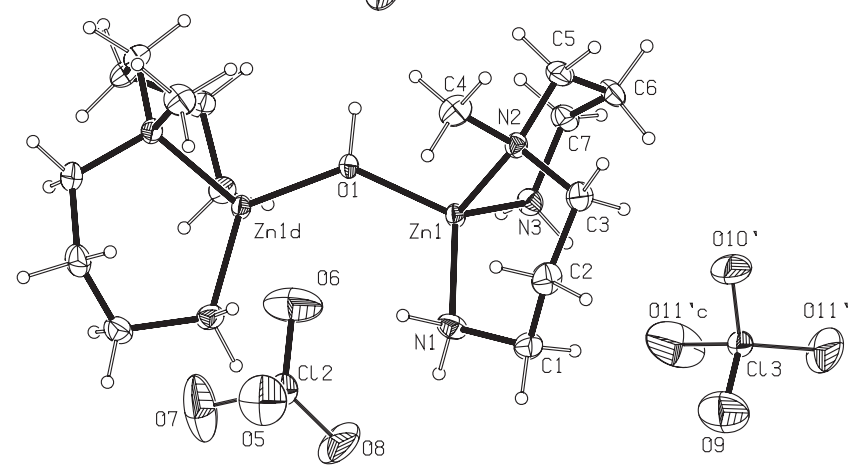

\begin{abstract}
$\mathrm{C}_{14} \mathrm{H}_{39} \mathrm{Cl}_{3} \mathrm{~N}_{6} \mathrm{O}_{13} \mathrm{Zn}_{2}$, monoclinic, $P 12_{1} / m 1$ (No. 11), $a=7.802(2) \AA, b=12.121(3) \AA, c=15.662(3) \AA, \beta=104.29(2)^{\circ}$, $V=1435.3 \AA^{3}, Z=2, R_{\mathrm{gt}}(F)=0.055, w R_{\mathrm{ref}}\left(F^{2}\right)=0.152, T=293 \mathrm{~K}$.
\end{abstract}

\section{Source of material}

All reagents and solvents were used as obtained without further purification. A solution of 5-methyl-1,4,9-triazanonane (mdipt) $(1 \mathrm{mmol}, 145 \mathrm{mg})$ in acetonitrile $(5 \mathrm{ml})$ was added to a stirred solution of zinc(II) perchlorate ( $1 \mathrm{mmol}, 370 \mathrm{mg}$ ) in water $(5 \mathrm{ml})$. A few drops of $0.1 \mathrm{M}$ solution of $\mathrm{HClO}_{4}$ in water was added to the above solution to adjust the $\mathrm{pH}=4-6$. Slow evaporation of the resulting solution in air for about a week, a large stack of colorless micro-crystals of $1,\left[(\operatorname{mtan}) \mathrm{Zn}\left(\mathrm{OH}_{2}\right)\right]\left(\mathrm{ClO}_{4}\right)_{2}$, were deposited and collected by filtration, washed with aqueous acetonitrile and dried in a vacuum desiccator over silica gel (yield 39\%). When the $\mathrm{pH}$ value of 1 was adjusted to about $\mathrm{pH} 7-11$ and was stood for two days, X-ray suitable colorless prism crystals of the title complex, $\left[(\operatorname{mtan}) \mathrm{Zn}\left(\mu_{2}-\mathrm{OH}\right) \mathrm{Zn}(\operatorname{mtan})\right]\left(\mathrm{ClO}_{4}\right)_{3}$, were deposited and collected by filtration, washed with aqueous acetonitrile and dried in a vacuum desiccator over silica gel (yield $88 \%$ ). Elemental analysis: found $-\mathrm{C}, 22.8 \% ; \mathrm{H}, 5.4 \% ; \mathrm{N}, 11.2 \% ; \mathrm{Cl}, 14.6 \% ; \mathrm{Zn}$, $17.6 \%$; calc. for $\mathrm{C}_{14} \mathrm{H}_{39} \mathrm{~N}_{6} \mathrm{Cl}_{3} \mathrm{O}_{13} \mathrm{Zn}_{2}-\mathrm{C}, 22.8 \%$; $\mathrm{H}, 5.3 \%$; , $11.4 \% ; \mathrm{Cl}, 14.4 \% ; \mathrm{Zn}, 17.8 \%$.

\section{Experimental details}

Several oxygen atoms of two perchlorate anions were disordered (s.o.f. $=0.5$ for $\mathrm{O} 7, \mathrm{O} 8$ attached to $\mathrm{Cl} 2$, and also s.o.f. $=0.5$ for $\mathrm{O} 10, \mathrm{O} 11, \mathrm{O} 10^{\prime}, \mathrm{O} 11^{\prime}$ attached to $\mathrm{Cl} 3$ ). One of the ordering arrangements is shown in the figure.

\section{Discussion}

Zinc-containing enzymes are ubiquitous in biology and constitute an essential class of metallo-proteins [1]. The study on the structure and property of zinc-site in zinc enzymes has been hot spots in the field of bioinorganic chemistry recently. Surprisingly, zinc-aqua model complexes have proven extremely difficult to stabilize, and all classical models have failed to stabilize a tetrahedral dicationic Zn-aqua species [2,3]. Previously [4], we reported an imdazolated-bridged dinuclear zinc complex, $\left[\mathrm{Zn}(2,3,2 \text {-tet })_{2}\left(\mu_{2}\right.\right.$-Im) $\mathrm{Zn}(2,3,2$-tet $\left.)\right]\left(\mathrm{ClO}_{4}\right)_{3}$ (2,3,2-tet is $1,4,8,11$-tetraazaundecane and Im is imidazolate anion), which is structurally similar to copper/zinc superoxide dismutase $\left(\mathrm{Cu}_{2}, \mathrm{Zn}_{2}-\mathrm{SOD}\right)$, however, as expected, has no any activity in catalyzing the dismutation of the superoxide ion $\left(\mathrm{O}_{2}^{-}\right)$to hydrogen peroxide and molecular oxygen.

The title complex is a discrete $\mathrm{OH}^{-}$bridged dinuclear zinc complex. The four-coordinate zinc ions in the complex exhibit a slightly distorted tetrahedral geometry. The average $\mathrm{Zn}-\mathrm{N}$ bond length $(2.024(2) \AA)$ is significantly shorter than that $(2.140 \AA)$ in $\left[\mathrm{Zn}_{2} \mathrm{~L}(\mathrm{OH})\left(\mathrm{NO}_{3}\right)_{2}\right][5,6]$. The $\mathrm{Zn}$-O bond length $(1.924(2) \AA)$ is shorter than those in zinc water complexes $(\sim 1.97 \AA)$, but significantly longer than those $(1.85 \AA, 1.86 \AA$ ) for the zinc hydroxide complexes in [3]. The $\mathrm{Zn}-\mathrm{Zn}$ distance (3.554(3) $\AA$ ) is close to those $(3.4 \AA-3.5 \AA)$ in several native enzymes. The $\mathrm{Zn}-\mathrm{O}-\mathrm{Zn}$ angle $\left(135.3(1)^{\circ}\right)$ is also comparable to those in literature for the enzymes Stenotrophomonas maltophilia and Bacteroides fragilis. One oxygen atom from a perchlorate and N(1) atom contributes to the formation of the hydrogen bond, with the hydrogen contact $d(\mathrm{~N} 1 \cdots \mathrm{H}-\mathrm{O} 2)=2.928(2) \AA$ and the angle $173.1^{\circ}$.

Table 1. Data collection and handling.

Crystal:

Wavelength:

$\mu$ :

Diffractometer, scan mode:

$2 \theta_{\max }$ :

$N(h k l)_{\text {measured }} N(h k l)_{\text {unique: }}$ Criterion for $I_{\mathrm{obs}}, N(h k l)_{\mathrm{gt}}$ :

$N(\text { param })_{\text {refined: }}$

Programs: colorless prism, size $0.30 \times 0.40 \times 0.50 \mathrm{~mm}$ Mo $K_{\alpha}$ radiation $(0.71073 \AA)$

$20.18 \mathrm{~cm}^{-1}$

Siemens R3m, $\omega$

$55^{\circ}$

3704,3458

$I_{\mathrm{obs}}>2 \sigma\left(I_{\mathrm{obs}}\right), 2387$

211

SHELXTL [7], SHELXTL-plus [8]

\footnotetext{
* Correspondence author (e-mail: hlzhu@wist.edu.cn)
} 
Table 2. Atomic coordinates and displacement parameters (in $\AA^{2}$ ).

\begin{tabular}{llllll}
\hline Atom & Site & $x$ & $y$ & $z$ & $U_{\text {iso }}$ \\
\hline $\mathrm{H}(1 \mathrm{H})$ & $2 e$ & 0.4596 & $1 / 4$ & 0.1971 & 0.08 \\
$\mathrm{H}(1)$ & $4 f$ & -0.0258 & 0.1112 & 0.2732 & 0.08 \\
$\mathrm{H}(2)$ & $4 f$ & 0.1637 & 0.1334 & 0.3606 & 0.08 \\
$\mathrm{H}(3)$ & $4 f$ & 0.1230 & -0.0117 & 0.1075 & 0.08 \\
$\mathrm{H}(4)$ & $4 f$ & 0.1464 & 0.0902 & 0.0673 & 0.08 \\
$\mathrm{H}(1 \mathrm{~A})$ & $4 f$ & 0.0439 & -0.0788 & 0.2869 & 0.08 \\
$\mathrm{H}(1 \mathrm{~B})$ & $4 f$ & 0.0118 & -0.0333 & 0.3745 & 0.08 \\
$\mathrm{H}(2 \mathrm{~A})$ & $4 f$ & 0.2587 & -0.1349 & 0.4177 & 0.08 \\
$\mathrm{H}(2 \mathrm{~B})$ & $4 f$ & 0.3245 & -0.0140 & 0.4245 & 0.08 \\
$\mathrm{H}(3 \mathrm{~A})$ & $4 f$ & 0.4960 & -0.1398 & 0.3597 & 0.08 \\
& & & & & \\
\hline
\end{tabular}

Table 2. Continued.

\begin{tabular}{lllrll}
\hline Atom & Site & $x$ & \multicolumn{1}{c}{$y$} & $U_{\text {iso }}$ \\
\hline $\mathrm{H}(3 \mathrm{~B})$ & $4 f$ & 0.3365 & -0.1432 & 0.2780 & 0.08 \\
$\mathrm{H}(4 \mathrm{~A})$ & $4 f$ & 0.6890 & 0.0122 & 0.3885 & 0.08 \\
$\mathrm{H}(4 \mathrm{~B})$ & $4 f$ & 0.6446 & 0.1235 & 0.3369 & 0.08 \\
$\mathrm{H}(4 \mathrm{C})$ & $4 f$ & 0.5340 & 0.0864 & 0.4029 & 0.08 \\
$\mathrm{H}(5 \mathrm{~A})$ & $4 f$ & 0.6525 & -0.0955 & 0.2538 & 0.08 \\
$\mathrm{H}(5 \mathrm{~B})$ & $4 f$ & 0.6408 & 0.0211 & 0.2123 & 0.08 \\
$\mathrm{H}(6 \mathrm{~A})$ & $4 f$ & 0.3835 & -0.1376 & 0.1511 & 0.08 \\
$\mathrm{H}(6 \mathrm{~B})$ & $4 f$ & 0.5415 & -0.1218 & 0.1087 & 0.08 \\
$\mathrm{H}(7 \mathrm{~A})$ & $4 f$ & 0.4317 & 0.0626 & 0.0722 & 0.08 \\
$\mathrm{H}(7 \mathrm{~B})$ & $4 f$ & 0.3194 & -0.0306 & 0.0173 & 0.08
\end{tabular}

Table 3. Atomic coordinates and displacement parameters (in $\AA^{2}$ ).

\begin{tabular}{|c|c|c|c|c|c|c|c|c|c|c|c|}
\hline Atom & Site & Occ. & $x$ & $y$ & $z$ & $U_{11}$ & $U_{22}$ & $U_{33}$ & $U_{12}$ & $U_{13}$ & $U_{23}$ \\
\hline $\mathrm{Zn}(1)$ & $4 f$ & & $0.26729(6)$ & $0.10338(4)$ & $0.22612(3)$ & $0.0394(2)$ & $0.0233(2)$ & $0.0344(2)$ & $0.0018(2)$ & $0.0117(2)$ & $0.0005(2)$ \\
\hline $\mathrm{O}(1)$ & $2 e$ & & $0.3595(6)$ & $1 / 4$ & $0.2236(3)$ & $0.047(2)$ & $0.021(2)$ & $0.080(3)$ & 0 & $0.031(2)$ & 0 \\
\hline $\mathrm{N}(1)$ & $4 f$ & & $0.0970(5)$ & $0.0845(3)$ & $0.3021(2)$ & $0.049(2)$ & $0.055(2)$ & $0.047(2)$ & $0.012(2)$ & $0.022(2)$ & $0.010(2)$ \\
\hline $\mathrm{N}(2)$ & $4 f$ & & $0.4698(4)$ & $0.0006(3)$ & $0.2885(2)$ & $0.038(2)$ & $0.030(2)$ & $0.043(2)$ & $0.002(1)$ & $0.006(1)$ & $0.003(2)$ \\
\hline $\mathrm{N}(3)$ & $4 f$ & & $0.2024(5)$ & $0.0395(3)$ & $0.1041(2)$ & $0.048(2)$ & $0.046(2)$ & $0.036(2)$ & $-0.000(2)$ & $0.010(2)$ & $-0.005(2)$ \\
\hline $\mathrm{C}(1)$ & $4 f$ & & $0.0900(6)$ & $-0.0306(4)$ & $0.3358(3)$ & $0.057(3)$ & $0.053(3)$ & $0.056(3)$ & $-0.007(2)$ & $0.026(2)$ & $-0.001(2)$ \\
\hline $\mathrm{C}(3)$ & $4 f$ & & $0.3984(6)$ & $-0.0977(4)$ & $0.3259(3)$ & $0.054(3)$ & $0.037(2)$ & $0.069(3)$ & $0.007(2)$ & $0.016(2)$ & $0.016(2)$ \\
\hline $\mathrm{C}(4)$ & $4 f$ & & $0.5955(6)$ & $0.0613(5)$ & $0.3605(3)$ & $0.051(3)$ & $0.068(3)$ & $0.047(3)$ & $-0.006(3)$ & $-0.003(2)$ & $0.003(3)$ \\
\hline $\mathrm{C}(5)$ & $4 f$ & & $0.5717(6)$ & $-0.0394(4)$ & $0.2252(3)$ & $0.040(2)$ & $0.047(3)$ & $0.067(3)$ & $0.011(2)$ & $0.018(2)$ & $0.006(2)$ \\
\hline$C(6)$ & $4 f$ & & $0.4643(6)$ & $-0.0841(4)$ & $0.1382(3)$ & $0.059(3)$ & $0.046(3)$ & $0.067(3)$ & $0.012(2)$ & $0.032(2)$ & $-0.009(2)$ \\
\hline $\mathrm{C}(7)$ & $4 f$ & & $0.3570(6)$ & $0.0005(4)$ & $0.0752(3)$ & $0.052(2)$ & $0.055(3)$ & $0.043(2)$ & $0.001(2)$ & $0.020(2)$ & $-0.009(2)$ \\
\hline $\mathrm{Cl}(1)$ & $2 e$ & & $0.7837(2)$ & $1 / 4$ & $0.1290(1)$ & $0.0399(8)$ & $0.0500(9)$ & $0.0555(9)$ & 0 & $0.0118(7)$ & 0 \\
\hline $\mathrm{O}(2)$ & $2 e$ & & $0.7667(9)$ & $1 / 4$ & $0.2189(4)$ & $0.129(5)$ & $0.074(4)$ & $0.085(4)$ & 0 & $0.065(4)$ & 0 \\
\hline $\mathrm{O}(3)$ & $2 e$ & & $0.6159(9)$ & $1 / 4$ & $0.0728(6)$ & $0.071(4)$ & $0.079(5)$ & $0.184(8)$ & 0 & $-0.052(5)$ & 0 \\
\hline $\mathrm{Cl}(2)$ & $2 e$ & & $0.1198(2)$ & $1 / 4$ & $0.5078(1)$ & $0.069(1)$ & $0.0487(9)$ & $0.0429(8)$ & 0 & $0.0168(8)$ & 0 \\
\hline $\mathrm{O}(5)$ & $2 e$ & & $0.170(1)$ & $1 / 4$ & $0.6008(3)$ & $0.161(7)$ & $0.151(7)$ & $0.059(4)$ & 0 & $0.018(4)$ & 0 \\
\hline $\mathrm{O}(6)$ & $2 e$ & & $0.2641(7)$ & $1 / 4$ & $0.4684(4)$ & $0.097(5)$ & $0.23(1)$ & $0.118(6)$ & 0 & $0.056(4)$ & 0 \\
\hline $\mathrm{O}(7)$ & $4 f$ & 0.50 & $0.059(1)$ & $0.3700(5)$ & $0.4903(6)$ & $0.36(2)$ & $0.063(7)$ & $0.135(9)$ & $0.02(1)$ & $0.13(1)$ & $0.011(7)$ \\
\hline $\mathrm{O}(8)$ & $4 f$ & 0.50 & $-0.0213(9)$ & $0.1873(7)$ & $0.4675(6)$ & $0.120(7)$ & $0.150(9)$ & $0.150(9)$ & $-0.088(6)$ & $0.044(7)$ & $-0.082(7)$ \\
\hline $\mathrm{Cl}(3)$ & $2 e$ & & $-0.0722(2)$ & $3 / 4$ & $0.1096(1)$ & $0.0422(8)$ & $0.0407(8)$ & $0.0557(9)$ & 0 & $0.0117(7)$ & 0 \\
\hline $\mathrm{O}(9)$ & $2 e$ & & $-0.1572(8)$ & $3 / 4$ & $0.1820(4)$ & $0.144(6)$ & $0.170(8)$ & $0.119(6)$ & 0 & $0.074(5)$ & 0 \\
\hline $\mathrm{O}(10)$ & $2 e$ & 0.50 & $-0.206(1)$ & $3 / 4$ & $0.0284(5)$ & $0.15(1)$ & $0.08(1)$ & $0.076(9)$ & 0 & $-0.032(9)$ & 0 \\
\hline $\mathrm{O}\left(10^{\prime}\right)$ & $2 e$ & 0.50 & $0.1148(8)$ & $3 / 4$ & $0.1488(8)$ & $0.045(6)$ & $0.10(1)$ & $0.23(2)$ & 0 & $0.034(9)$ & 0 \\
\hline $\mathrm{O}(11)$ & $4 f$ & 0.50 & $0.042(1)$ & $-0.3360(9)$ & $0.1142(8)$ & $0.34(2)$ & $0.18(1)$ & $0.31(2)$ & $0.21(1)$ & $0.03(2)$ & $-0.03(1)$ \\
\hline $\mathrm{O}\left(11^{\prime}\right)$ & $4 f$ & 0.50 & $-0.114(1)$ & $-0.3524(7)$ & $0.0665(7)$ & $0.18(1)$ & $0.152(8)$ & $0.22(1)$ & $-0.094(8)$ & $0.104(8)$ & $-0.142(8)$ \\
\hline
\end{tabular}

Acknowledgments. The authors thank the Education Office of Hubei Province, P. R. China, for the research grant No. 2002B29002 and the Natural Science Foundation of Hubei Province, P. R. China, for the research grant No. 2003ABB010.

\section{References}

1. Lipscomb, W. N.; Strater, N.: Recent Advances in Zinc Enzymology. Chem. Rev. 96 (1996) 2375-2433.

2. Bergquist, C.; Parkin, G.: Protonation of the Hydroxide Ligand in a Synthetic Analogue of Carbonic Anhydrase, $\left[\mathrm{Tp}^{\mathrm{But}, \mathrm{Me}}\right] \mathrm{ZnOH}$ : Inhibition of Reactivity Towards $\mathrm{CO}_{2}$. J. Am. Chem. Soc. 121 (1999) 6322-6323.

3. Kimblin, C.; Allen, W. E.; Parkin, G.: The synthesis and structure of PimPri,But $] \mathrm{ZnOH}\left(\mathrm{ClO}_{4}\right)$ : a tris(imidazolyl)phosphine zinc hydroxide complex and a proposed structural model for carbonic anhydrase. J. Chem. Soc., Chem. Commun. (1995) 1813-1815.

4. Zhu, H.-L.; Tong, Y.-X.; Zhao, J.; Duan, C.-Y.; Chen, X.-M.; Tang, W.-X.: Crystal Structure and Characterization of [Bis $N^{\prime}$-bis(2-aminoethyl)propane-1,3-diamine(-imidazolato- $N, N^{\prime}$ )-dizinc(II)] Triperchlorate. Aust. J. Chem. 52 (1999) 709-711.
5. Kaminskaia, N. V.; Springler, B.; Lippard, S. J.: Hydrolysis of baita-Lactam Antibiotics Catalyzed by Dinuclear Zinc(II) Complexes: Functional Mimics of Metallo-baita-lactamases J. Am. Chem. Soc. 122 (2000) 6411-6422.

6. Kaminskaia, N. V.; Spingler, B.; Lippard, S. J.: Intermediate in baita-Lactam Hydrolysis Catalyzed by a Dinuclear Zinc(II) Complex: Relevance to the Mechanism of Metallo-baita-lactamase. J. Am. Chem. Soc. 123 (2001) 6555-6563.

7. Sheldrick, G. M.: Siemens SHELXTL (Version 5.0): Siemens Industrial Automation, Inc., Analytical Instrumentation, USA 1995.

8. Sheldrick, G. M.: SHELXTL-plus. Release 4.1. Siemens Analytical X-ray Instruments Inc., Madison Wisconsin, USA 1991. 